\title{
HAUSDORFF DIMENSION OF THE LARGE VALUES OF WEYL SUMS
}

\author{
CHANGHAO CHEN AND IGOR E. SHPARLINSKI
}

\begin{abstract}
The authors have recently obtained a lower bound of the Hausdorff dimension for the sets of vectors $\left(x_{1}, \ldots, x_{d}\right) \in$ $[0,1)^{d}$ with large Weyl sums, namely of vectors for which

$$
\left|\sum_{n=1}^{N} \exp \left(2 \pi i\left(x_{1} n+\ldots+x_{d} n^{d}\right)\right)\right| \geqslant N^{\alpha}
$$

for infinitely many integers $N \geqslant 1$. Here we obtain an upper bound for the Hausdorff dimension of these exceptional sets.
\end{abstract}

\section{INTRODUCTION}

1.1. Motivation and background. For an integer $d \geqslant 2$, let $\mathrm{T}_{d}=$ $(\mathbb{R} / \mathbb{Z})^{d}$ be the $d$-dimensional unit torus.

For a vector $\mathbf{x}=\left(x_{1}, \ldots, x_{d}\right) \in \mathrm{T}_{d}$ and $N \in \mathbb{N}$, we consider the exponential sums

$$
S_{d}(\mathbf{x} ; N)=\sum_{n=1}^{N} \mathbf{e}\left(x_{1} n+\ldots+x_{d} n^{d}\right),
$$

which are commonly called Weyl sums, where throughout the paper we denote $\mathbf{e}(x)=\exp (2 \pi i x)$.

The authors [2, Appendix A] have shown that for almost all $\mathbf{x} \in \mathrm{T}_{d}$ (with respect to Lebesgue measure) one has

$$
\left|S_{d}(\mathbf{x} ; N)\right| \leqslant N^{1 / 2+o(1)} \text { as } N \rightarrow \infty,
$$

see also [3, Theorem 2.1] for a different proof. It is very natural to conjecture that the exponent $1 / 2$ is the best possible value, and indeed for $d=2$ the authors [4] proved that for almost all $\left(x_{1}, x_{2}\right) \in \mathrm{T}_{2}$ we have

$$
\limsup _{N \rightarrow \infty}\left|S_{2}\left(\left(x_{1}, x_{2}\right) ; N\right)\right| N^{-1 / 2}(\log \log N)^{-1 / 6}=\infty .
$$

However there seems to be no results in this direction for $d \geqslant 3$.

2010 Mathematics Subject Classification. 11L15, 28A78, 28A80.

Key words and phrases. Weyl sums, Hausdorff dimension. 
For integer $d \geqslant 2$ and $0<\alpha<1$ our main object is defined as

$$
\mathcal{E}_{d, \alpha}=\left\{\mathbf{x} \in \mathrm{T}_{d}:\left|S_{d}(\mathbf{x} ; N)\right| \geqslant N^{\alpha} \text { for infinitely many } N \in \mathbb{N}\right\} .
$$

We can restate the bound (1.1) in the following way: for any $\alpha \in$ $(1 / 2,1)$ the set $\mathcal{E}_{d, \alpha}$ is of Lebesgue measure zero. Here we are mostly interested in the structure of the sets $\mathcal{E}_{d, \alpha}$, and for convenience we call the set $\mathcal{E}_{d, \alpha}$ the exceptional set for any integer $d \geqslant 2$ and each $0<\alpha<1$.

The authors [2] show that in terms of the Baire categories and Hausdorff dimension the exceptional sets $\mathcal{E}_{d, \alpha}$ are quite massive. By [2, Theorem 1.3], for each $0<\alpha<1$ and integer $d \geqslant 2$ the set $\mathrm{T}_{d} \backslash \mathcal{E}_{d, \alpha}$ is of the first Baire category. Alternatively, this is equivalent to the statement that the complement $\mathrm{T}_{d} \backslash \Xi_{d}$ to the set

$$
\begin{aligned}
\Xi_{d}=\left\{\mathrm{x} \in \mathrm{T}_{d}: \forall \varepsilon>0,\left|S_{d}(\mathbf{x} ; N)\right|\right. & \geqslant N^{1-\varepsilon} \\
& \text { for infinitely many } N \in \mathbb{N}\}
\end{aligned}
$$

is of first category, see [2] for more details and reference therein. For the Hausdorff dimension it is shown in [2, Theorem 1.5] that for any $d \geqslant 2$ and $0<\alpha<1$ one has

$$
\operatorname{dim} \mathcal{E}_{d, \alpha} \geqslant \xi(d, \alpha)>0
$$

with some explicit constant $\xi(d, \alpha)$.

We remark that the authors [3, Corollary 1.9] have obtained a nontrivial upper bound for the Hausdorff dimension of $\mathcal{E}_{d, \alpha}$ for some $\alpha$, however the bounds there are not fully explicit and do not cover the whole range $1 / 2<\alpha<1$.

Here we obtain the nontrivial upper bound of $\operatorname{dim} \mathcal{E}_{d, \alpha}$ for all $1 / 2<$ $\alpha<1$ and $d \geqslant 2$.

On the other hand, we note that we do not have any plausible conjecture about the exact value of the Hausdorff dimension of $\mathcal{E}_{d, \alpha}$.

1.2. Main results. For $\mathcal{A} \subseteq \mathbb{R}^{d}$, the $s$-dimension Hausdorff measure of $\mathcal{A}$ is defined as

$$
\mathcal{H}^{s}(\mathcal{A})=\lim _{\delta \rightarrow 0} \mathcal{H}_{\delta}^{s}(\mathcal{A})
$$

where

$$
\mathcal{H}_{\delta}^{s}(\mathcal{A})=\inf \left\{\sum_{i=1}^{\infty}\left(\operatorname{diam} \mathcal{U}_{i}\right)^{s}: \mathcal{A} \subseteq \bigcup_{i=1}^{\infty} \mathcal{U}_{i} \text { and } \operatorname{diam} \mathcal{U}_{i} \leqslant \delta, i \in \mathbb{N}\right\}
$$

The Hausdorff dimension of $\mathcal{A}$ is defined as

$$
\begin{aligned}
\operatorname{dim} \mathcal{A} & =\inf \left\{s>0: \mathcal{H}^{s}(\mathcal{A})=0\right\} \\
& =\sup \left\{s>0: \mathcal{H}^{s}(\mathcal{A})=\infty\right\} .
\end{aligned}
$$


We refer to [5] for more details and properties of Hausdorff dimension.

For integer $d \geqslant 2$ and $0<\alpha<1$ denote

$$
\mathfrak{u}(d, \alpha)=\min _{k=0, \ldots, d-1} \frac{\left(2 d^{2}+4 d\right)(1-\alpha)+k(k+1)}{4-2 \alpha+2 k} .
$$

Theorem 1.1. For any integer $d \geqslant 2$ and $0<\alpha<1$ we have

$$
\operatorname{dim} \mathcal{E}_{d, \alpha} \leqslant \mathfrak{u}(d, \alpha) .
$$

For $d \geqslant 2$ and any $1 / 2<\alpha<1$ an elementary calculation gives that $\mathfrak{u}(d, \alpha)<d$. In fact by taking $k=d-1$ in (1.4) we derive

$$
\mathfrak{u}(d, \alpha) \leqslant d-\frac{d(d+1)(2 \alpha-1)}{2(d+1-\alpha)} .
$$

Thus, we have

Corollary 1.2. For any integer $d \geqslant 2$ and any $1 / 2<\alpha<1$ we have $\operatorname{dim} \mathcal{E}_{d, \alpha}<d$.

Furthermore taking, for example, $k=0$ in (1.4) we obtain

$$
\operatorname{dim} \mathcal{E}_{d, \alpha} \leqslant \mathfrak{u}(d, \alpha) \leqslant \frac{\left(2 d^{2}+4 d\right)(1-\alpha)}{4-2 \alpha} .
$$

We note that although the lower bound (1.3) and the upper bound of Theorem 1.1 are of very different magnitude with respect to $d$, however for $\alpha \rightarrow 1$ they give the same rate of convergency to zero which of order $1-\alpha$. More precisely, the explicit formula for $\xi(d, \alpha)$ from [2] and the formula (1.4) yield

$$
c_{1}(d) \leqslant \liminf _{\alpha \rightarrow 1}(1-\alpha)^{-1} \operatorname{dim} \mathcal{E}_{d, \alpha} \leqslant \limsup _{\alpha \rightarrow 1}(1-\alpha)^{-1} \operatorname{dim} \mathcal{E}_{d, \alpha} \leqslant c_{2}(d)
$$

for two positive constants $c_{1}(d), c_{2}(d)$ depending only on $d$. In fact for $d=2$ we have

$$
c_{1}(2)=3 \quad \text { and } \quad c_{2}(2)=8,
$$

while for $d \geqslant 3$ we have

$$
c_{1}(d)=\max _{\nu=1, \ldots, d} \min \left\{\frac{1}{\nu}, \frac{2}{2 d-\nu}\right\} \quad \text { and } \quad c_{2}(d)=d^{2}+2 d .
$$

In particular, we have

Corollary 1.3. For any integer $d \geqslant 2$, if $\alpha \rightarrow 1$ then $\operatorname{dim} \mathcal{E}_{d, \alpha} \rightarrow 0$.

From the definition of $\Xi_{d}$, see (1.2), we have $\Xi_{d} \subseteq \mathcal{E}_{d, \alpha}$ for any $0<\alpha<1$. Therefore

Corollary 1.4. For and integer $d \geqslant 2$, we have $\operatorname{dim} \Xi_{d}=0$. 


\section{Preliminaries}

2.1. Notation and conventions. Throughout the paper, the notation $U=O(V), U \ll V$ and $V \gg U$ are equivalent to $|U| \leqslant c|V|$ for some positive constant $c$, which throughout the paper may depend on the degree $d$ and occasionally on the small real positive parameters $\varepsilon$ and $\delta$.

For any quantity $V>1$ we write $U=V^{o(1)}$ (as $V \rightarrow \infty$ ) to indicate a function of $V$ which satisfies $|U| \leqslant V^{\varepsilon}$ for any $\varepsilon>0$, provided $V$ that is large enough.

We use $\# \mathcal{X}$ to denote the cardinality of set $\mathcal{X}$.

We always identify $\mathrm{T}_{d}$ with half-open unit cube $[0,1)^{d}$, in particular we naturally associate Euclidean norm $\|x\|$ with points $x \in \mathrm{T}_{d}$. Moreover we always assume that $d \geqslant 2$.

We say that some property holds for almost all $\mathbf{x} \in \mathrm{T}_{d}$ if it holds for a set $\mathcal{X} \subseteq \mathrm{T}_{d}$ of Lebesgue measure $\lambda(\mathcal{X})=1$.

We always keep the subscript $d$ in notations for our main objects of interest such as $\mathcal{E}_{d, \alpha}, S_{d}(\mathbf{x} ; N)$ and $\mathrm{T}_{d}$, but sometimes suppress it in auxiliary quantities.

2.2. Mean value theorems. The Vinogradov mean value theorem in the currently known form, due to Bourgain, Demeter and Guth [1] for $d \geqslant 4$ and Wooley [7] for $d=3$, asserts that,

$$
\int_{\mathbf{T}_{d}}\left|S_{d}(\mathbf{x} ; N)\right|^{2 s(d)} d \mathbf{x} \leqslant N^{s(d)+o(1)}
$$

where $s(d)=d(d+1) / 2$. We will use the following result due to Wooley [9, Theorem 1.1], which extends the bound to the Weyl sums with weights.

Lemma 2.1. For any sequence of complex weights $\mathbf{a}=\left(a_{n}\right)_{n=1}^{\infty}$, and any integer $N \geqslant 1$, we have the upper bound

$$
\int_{\mathrm{T}_{d}}\left|\sum_{n=1}^{N} a_{n} \mathbf{e}\left(x_{1} n+\ldots+x_{d} n^{d}\right)\right|^{2 s(d)} d \mathbf{x} \leqslant N^{o(1)}\left(\sum_{n=1}^{N}\left|a_{n}\right|^{2}\right)^{s(d)} .
$$

2.3. Completion method. The following bound is special case of $[3$, Lemma 3.2], and for completeness we give a proof here.

Lemma 2.2. For $\mathrm{x} \in \mathrm{T}_{d}$ and $1 \leqslant M \leqslant N$ we have

$$
S_{d}(\mathbf{x} ; M) \ll W_{d}(\mathbf{x} ; N),
$$


where

$$
W_{d}(\mathbf{x} ; N)=\sum_{h=1}^{N} \frac{1}{h}\left|\sum_{n=1}^{N} \mathbf{e}(h n / N) \mathbf{e}\left(x_{1} n+\ldots+x_{d} n^{d}\right)\right| .
$$

Proof. For $\mathbf{x} \in \mathrm{T}_{d}$ and $n \in \mathbb{N}$ denote

$$
f(n)=x_{1} n+\ldots+x_{d} n^{d} .
$$

Observe that by the orthogonality

$$
\frac{1}{N} \sum_{h=1}^{N} \sum_{k=1}^{M} \mathbf{e}(h(n-k) / N)= \begin{cases}1 & n=1, \ldots, M \\ 0 & \text { otherwise. }\end{cases}
$$

We also note that for $1 \leqslant h, M \leqslant N$ we have

$$
\sum_{k=1}^{M} \mathbf{e}(h k / N) \ll \frac{N}{\min \{h, N+1-h\}},
$$

see [6, Equation (8.6)]. It follows that

$$
\begin{aligned}
S_{d}(\mathbf{x} ; M) & =\sum_{n=1}^{N} \mathbf{e}(f(n)) \frac{1}{N} \sum_{h=1}^{N} \sum_{k=1}^{M} \mathbf{e}(h(n-k) / N) \\
& =\frac{1}{N} \sum_{h=1}^{N} \sum_{k=1}^{M} \mathbf{e}(-h k / N) \sum_{n=1}^{N} \mathbf{e}(f(n)) \mathbf{e}(h n / N) \\
& \ll \sum_{h=1}^{N} \frac{1}{\min \{h, N+1-h\}}\left|\sum_{n=1}^{N} \mathbf{e}(h n / N) \mathbf{e}(f(n))\right| \\
& \ll \sum_{h=1}^{N} \frac{1}{h}\left|\sum_{n=1}^{N} \mathbf{e}(h n / N) \mathbf{e}(f(n))\right|
\end{aligned}
$$

which finishes the proof.

Observe that for any $N$ there exists a sequence $b_{N}(n), n=1, \ldots, N$ such that

$$
b_{N}(n) \ll \log N, \quad n=1, \ldots, N,
$$

and $W_{d}(\mathbf{x} ; N)$ can be written as

$$
W_{d}(\mathbf{x} ; N)=\sum_{n=1}^{N} b_{N}(n) \mathbf{e}\left(x_{1} n+\ldots+x_{d} n^{d}\right) .
$$

From Lemma 2.2 we immediately obtain: 
Corollary 2.3. Let $0<\alpha<1$ and $N_{i}=2^{i}, i \in \mathbb{N}$. Using above notation for any $\eta>0$ we have

$$
\mathcal{E}_{d, \alpha+\eta} \subseteq\left\{\mathbf{x} \in \mathrm{T}_{d}:\left|W_{d}\left(\mathbf{x} ; N_{i}\right)\right| \geqslant N_{i}^{\alpha} \text { for infinitely many } i \in \mathbb{N}\right\} .
$$

Thus for the purpose of estimate the set $\mathcal{E}_{d, \alpha}$ it is sufficient to know the size of the set

$$
\left\{\mathbf{x} \in \mathrm{T}_{d}:\left|W_{d}(\mathbf{x} ; N)\right| \geqslant N^{\alpha}\right\},
$$

which we investigate in Section 2.4 below.

2.4. Distribution of large values of exponential sums. We first remark that the results in this subsection are special forms of [3], see also [8, Lemma 2.1]. For completeness we give proofs for these special cases.

For $\mathbf{u} \in \mathbb{R}^{d}$ and $\boldsymbol{\zeta}=\left(\zeta_{1}, \ldots, \zeta_{d}\right)$ with $\zeta_{j}>0, j=1, \ldots, d$, we define the $d$-dimensional rectangle (or box) with the centre $\mathbf{u}$ and the side lengths $2 \boldsymbol{\zeta}$ by

$$
\mathcal{R}(\mathbf{u}, \boldsymbol{\zeta})=\left[u_{1}-\zeta_{1}, u_{1}+\zeta_{1}\right) \times \ldots \times\left[u_{d}-\zeta_{d}, u_{d}+\zeta_{d}\right) .
$$

In analogue of [8, Lemma 2.1] and [3, Lemma 3.5] we obtain:

Lemma 2.4. Let $0<\alpha<1$ and let $\varepsilon>0$ be sufficiently small. If $\left|W_{d}(\mathbf{x} ; N)\right| \geqslant N^{\alpha}$ for some $\mathbf{x} \in \mathrm{T}_{d}$, then

$$
\left|W_{d}(\mathbf{y} ; N)\right| \geqslant N^{\alpha} / 2
$$

holds for any $\mathbf{y} \in \mathcal{R}(\mathbf{x}, \boldsymbol{\zeta})$ provided that $N$ is large enough and

$$
0<\zeta_{j} \leqslant N^{\alpha-j-1-\varepsilon}, \quad j=1, \ldots, d .
$$

Proof. For any $h=1, \ldots, N$ we have

$$
\begin{gathered}
\sum_{n=1}^{N} \mathbf{e}(h n / N)\left(\mathbf{e}\left(x_{1} n+\ldots+x_{d} n^{d}\right)-\mathbf{e}\left(y_{1} n+\ldots+y_{d} n^{d}\right)\right) \\
\ll \sum_{n=1}^{N} \sum_{j=1}^{d} \zeta_{j} n^{j} \leqslant N^{\alpha-\varepsilon / 2} .
\end{gathered}
$$

The last estimate holds for all large enough $N$. By Lemma 2.2 we obtain

$$
\left|W_{d}(\mathbf{x} ; N)-W_{d}(\mathbf{y} ; N)\right| \ll N^{\alpha-\varepsilon / 2} \log N \leqslant N^{\alpha} / 2,
$$

which holds for all large enough $N$ and gives the result.

In analogue of [3, Lemma 3.7] from Lemmas 2.2 and 2.4 we obtain: 
Lemma 2.5. Let $0<\alpha<1$ and $\varepsilon>0$ be a small parameter. For each $j=1, \ldots, d$ let

$$
\zeta_{j}=1 /\left\lceil N^{j+1+\varepsilon-\alpha}\right\rceil .
$$

We divide $\mathrm{T}_{d}$ into

$$
U=\prod_{j=1}^{d} \zeta_{j}^{-1}
$$

boxes of the type

$$
\left[n_{1} \zeta_{1},\left(n_{1}+1\right) \zeta_{1}\right) \times \ldots \times\left[n_{d} \zeta_{d},\left(n_{d}+1\right) \zeta_{d}\right)
$$

where $n_{j}=0, \ldots, 1 / \zeta_{j}-1, j=1, \ldots, d$. Let $\mathfrak{R}$ be the collection of these boxes, and

$$
\widetilde{\mathfrak{R}}=\left\{\mathcal{R} \in \mathfrak{R}: \exists \mathbf{x} \in \mathcal{R} \text { with }\left|W_{d}(\mathbf{x} ; N)\right| \geqslant N^{\alpha}\right\} .
$$

Then one has

$$
\# \widetilde{\mathfrak{R}} \leqslant U N^{s(d)(1-2 \alpha)+o(1)} .
$$

Proof. Let $\mathcal{R} \in \mathfrak{R}$. By Lemma 2.4 if $\left|W_{d}(\mathbf{x} ; N)\right| \geqslant N^{\alpha}$ for some $\mathbf{x} \in \mathcal{R}$, then for any $\mathbf{y} \in \mathcal{R}$ we have $\left|W_{d}(\mathbf{y} ; N)\right| \geqslant N^{\alpha} / 2$. Combining with Lemma 2.1 and (2.1), (2.2) we derive

$$
N^{2 s(d) \alpha} \# \widetilde{\Re} \prod_{j=1}^{d} \zeta_{j} \ll \int_{\mathrm{T}_{d}}\left|W_{d}(\mathbf{x} ; N)\right|^{2 s(d)} d \mathbf{x} \leqslant N^{s(d)+o(1)},
$$

which yields the desired bound.

Note that the above bound of $\# \widetilde{\mathfrak{R}}$ is nontrivial when $1 / 2<\alpha<1$.

From Corollary 2.3 and Lemma 2.5 we formulate the following Corollary 2.6 for the convenience of our applications.

Corollary 2.6. Let $0<\alpha<1$ and $N_{i}=2^{i}, i \in \mathbb{N}$. Then for any $\eta>0$ we have

$$
\mathcal{E}_{d, \alpha+\eta} \subseteq \bigcap_{k=1}^{\infty} \bigcup_{i=k}^{\infty} \bigcup_{\mathcal{R} \in \mathfrak{R}(i)} \mathcal{R}
$$

where each $\mathcal{R}$ of $\mathfrak{R}(i)$ has the side length $\boldsymbol{\zeta}=\left(\zeta_{1}, \ldots, \zeta_{d}\right)$ such that

$$
\zeta_{j}=1 /\left\lceil N_{i}^{j+1+\varepsilon-\alpha}\right\rceil, \quad j=1, \ldots, d,
$$

and furthermore

$$
\# \mathfrak{R}(i) \leqslant N_{i}^{s(d)-2 \alpha s(d)} \prod_{j=1}^{d} \zeta_{j}^{-1} \leqslant N_{i}^{2 s(d)(1-\alpha)+d(1-\alpha)+d \varepsilon+o(1)} .
$$




\section{Proof of Theorem 1.1}

We start from some auxiliary results. First, we adapt the definition of the singular value function from [5, Chapter 9] to the following.

Definition 3.1. Let $\mathcal{R} \subseteq \mathbb{R}^{d}$ be a rectangle with side lengths

$$
r_{1} \geqslant \ldots \geqslant r_{d}
$$

For $0<t \leqslant d$ we set

$$
\varphi_{0, t}(\mathcal{R})=r_{1}^{t}
$$

and for $k=1, \ldots, d-1$ we define

$$
\varphi_{k, t}(\mathcal{R})=r_{1} \ldots r_{k} r_{k+1}^{t-k}
$$

Note that for a rectangle $\mathcal{R} \subseteq \mathbb{R}^{2}$ with the side length $r_{1} \geqslant r_{2}$ we have

$$
\varphi_{k, t}(\mathcal{R})= \begin{cases}r_{1}^{t} & \text { for } k=0 \\ r_{1} r_{2}^{t-1} & \text { for } k=1\end{cases}
$$

Remark 3.2. The notation $\varphi_{k, t}(\mathcal{R})$ roughly means that we can cover the rectangle $\mathcal{R}$ by about (up to a constant factor)

$$
\frac{r_{1}}{r_{k+1}} \ldots \frac{r_{k}}{r_{k+1}}
$$

balls of radius $r_{k+1}$, and hence this leads to the term

$$
\varphi_{k, t}(\mathcal{R})=\frac{r_{1}}{r_{k+1}} \ldots \frac{r_{k}}{r_{k+1}} r_{k+1}^{t}
$$

in the expression for the Hausdorff measure with the parameter $t$ (again up to a constant factor which does not affect our results).

From the definition of the Hausdorff dimension, using the above notation, we have the following inequality

$$
\begin{aligned}
\operatorname{dim} \mathcal{E}_{d, \alpha+\varepsilon} \leqslant \inf \left\{t>0: \sum_{i=1}^{\infty} \sum_{\mathcal{R} \in \Re(i)} \varphi_{k, t}(\mathcal{R})<\infty\right. \\
\text { for some } k=0, \ldots, d-1\} .
\end{aligned}
$$


Now we turn to the proof of Theorem 1.1. For $k=1, \ldots, d-1$ and $0<t \leqslant d$ we have

$$
\begin{aligned}
& \sum_{\mathcal{R} \in \mathfrak{R}(i)} \varphi_{k, t}(\mathcal{R})=\# \Re(i) \zeta_{k+1}^{t-k} \prod_{j=1}^{k} \zeta_{j} \\
& \leqslant N_{i}^{2 s(d)(1-\alpha)+d(1-\alpha)+d \varepsilon+o(1)} \\
& \times\left(N_{i}^{\alpha-1-\varepsilon-(k+1)}\right)^{t-k} \prod_{j=1}^{k} N_{i}^{\alpha-j-1-\varepsilon} \\
& \leqslant N_{i}^{2 s(d)(1-\alpha)+d(1-\alpha)+d \varepsilon+(t-k)(\alpha-k-2-\varepsilon)+k(\alpha-1-\varepsilon)-s(k)+o(1) .}
\end{aligned}
$$

Here and in the following we denote

$$
s(k)=\frac{k(k+1)}{2} .
$$

We remark that (3.2) also holds for the case $k=0$, in which we have $s(k)=0$. To be precise for $k=0$ we have

$$
\sum_{\mathcal{R} \in \mathfrak{R}(i)} \varphi_{0, t}(\mathcal{R}) \leqslant N_{i}^{2 s(d)(1-\alpha)+d(1-\alpha)+d \varepsilon+t(\alpha-2-\varepsilon)+o(1)} .
$$

Applying (3.1) we conclude that

$$
\operatorname{dim} \mathcal{E}_{d, \alpha+\eta} \leqslant t
$$

provided that the parameters $\alpha, \rho, k, t$ satisfy the following further condition

$$
2 s(d)(1-\alpha)+d(1-\alpha)+(t-k)(\alpha-k-2)+k(\alpha-1)-s(k)<0,
$$

which becomes

$$
t>\frac{2 s(d)(1-\alpha)+d(1-\alpha)+s(k)}{k+2-\alpha} .
$$

By the arbitrary choice of $\eta$ we finish the proof.

\section{ACKNOWLEDGEMENT}

This work was supported by ARC Grant DP170100786.

\section{REFERENCES}

[1] J. Bourgain, C. Demeter and L. Guth, 'Proof of the main conjecture in Vinogradov's mean value theorem for degrees higher than three', Ann. Math., 184 (2016), 633-682. 4

[2] C. Chen and I. E. Shparlinski, 'On large values of Weyl sums', Preprint, 2019, available at https://arxiv.org/abs/1901.01551.1, 2, 3

[3] C. Chen and I. E. Shparlinski, 'New bounds of Weyl sums', Preprint, 2019, available at https://arxiv.org/abs/1903.07330.1, 2, 4, 6 
[4] C. Chen and I. E. Shparlinski, 'Large and small values of quadratic Weyl sums', Preprin, 2019, available at https://arxiv.org/abs/1907.03101. 1

[5] K. J. Falconer, Fractal geometry: Mathematical foundations and applications, John Wiley, 2nd Ed., 2003. 2, 8

[6] H. Iwaniec and E. Kowalski, Analytic number theory, Amer. Math. Soc., Providence, RI, 2004. 5

[7] T. D. Wooley, 'The cubic case of the main conjecture in Vinogradov's mean value theorem', Adv. in Math., 294 (2016), 532-561. 4

[8] T. D. Wooley, 'Perturbations of Weyl sums', Internat. Math. Res. Notices, 2016 (2016), 2632-2646. 6

[9] T. D. Wooley, 'Nested efficient congruencing and relatives of Vinogradov's mean value theorem', Proc. London Math. Soc., (to appear). 4

Department of Pure Mathematics, University of New South Wales, Sydney, NSW 2052, Australia

E-mail address: changhao.chenm@gmail.com

Department of Pure Mathematics, University of New South Wales, Sydney, NSW 2052, Australia

E-mail address: igor.shparlinski@unsw.edu.au 